\title{
Kernos
}

Revue internationale et pluridisciplinaire de religion grecque antique

3| 1990

Varia

\section{Les oracles de Léon VI le Sage, leurs origines et leur postérité}

\section{André Deisser}

\section{(2) OpenEdition \\ Journals}

\section{Édition électronique}

URL : http://journals.openedition.org/kernos/980

DOI : $10.4000 /$ kernos.980

ISSN : 2034-7871

\section{Éditeur}

Centre international d'étude de la religion grecque antique

\section{Édition imprimée}

Date de publication : 1 janvier 1990

ISSN : 0776-3824

\section{Référence électronique}

André Deisser, "Les oracles de Léon VI le Sage, leurs origines et leur postérité », Kernos [En ligne], 3 | 1990, mis en ligne le 19 avril 2011, consulté le 30 avril 2019. URL : http://journals.openedition.org/ kernos/980 ; DOI : 10.4000/kernos.980 


\section{LES ORACLES DE LÉON VI LE SAGE, LEURS ORIGINES ET LEUR POSTÉRITÉ}

Le poète néo-latin Hugo Favolius rapporte qu'à la fin du mois de juin 1545 , un ambassadeur de Charles Quint se présenta, à bord d'une galère vénitienne, devant le port de Raguse. Quand le Recteur de cette République reconnut, sur les bâtons de proue, les pavillons marqués de trois lions rampants (triplices atroci horrore leones) et les aigles à deux têtes de l'Empire (geminas aquilas), lui revinrent à l'esprit, selon Favolius, d'anciennes prophéties qu'il développa dans un ample monologue : d'antiques oracles auraient promis qu'un personnage impérial viendrait libérer les Balkans, Constantinople et l'Asie Mineure de l'oppression des Turcs. À son arrivée, reverdirait une Montagne Magique à présent dénudée. Bref, dans l'empire universel enfin constitué, «la justice, les douceurs de la paix, le loisir après la fin des combats surgiront pour toujours (...) et orneront les villes florissantes en y faisant régner leurs lois». Bien mieux, le Recteur de Raguse proclama que c'est Charles Quint en personne qui a été désigné du fond des âges pour ce rôle ${ }^{1}$. Et ceci n'est pas uniquement une rêverie particulière à notre poète, mais c'est l'écho en vers latins de toute une littérature, savante ou populaire, qui l'a précédé. Cependant, aux environs de 1535, l'alliance entre les Turcs et les Français porta un coup décisif à ce projet d'une croisade menée par l'ensemble des pays chrétiens $^{2}$. C'est alors qu'il apparut à certains que Charles Quint, à lui seul, pourrait se présenter comme un libérateur providentiel. En effet, dès 1525 , juste après la bataille de Pavie, l'humaniste grec Janus Lascaris l'avait personnellement exhorté à la guerre non plus contre les Français, mais contre les Turcs ${ }^{3}$. Vers le même temps, Arsène, archevêque de Monembasie, avait publié à cinq cents exemplaires une lettre ouverte rédigée dans ce sens et destinée à un souverain anonyme,

1 Hugo Favolius, Hodoeporicum Byzantinum, Louvain, 1563, f. 16-25.

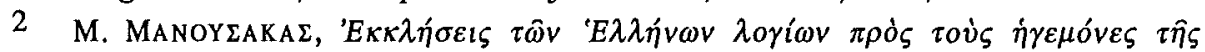

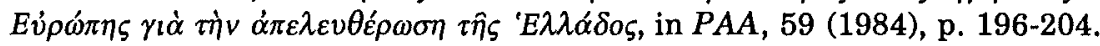

3 B. KNös, Un ambassadeur de l'hellénisme, Janus Lascaris et la tradition grécobyzantine dans l'humanisme français, Uppsala-Paris, 1945, p. 186-191 et M. MANOYEAKAL, art. cit., p. 203, qui apporte un complément bibliographique. 
mais qui ne pouvait être que Charles Quint, ainsi que l'a démontré Manoussos Manoussacas 4 .

Même quand les intellectuels grecs se turent après 1535 (découragés par l'alliance franco-turque), des traditions populaires n'en continuèrent pas moins d'entretenir l'espérance que la puissance ottomane allait bientôt s'écrouler au profit de l'empereur d'Occident ${ }^{5}$. Le témoignage le plus curieux en est un poème de plus de mille vers, rédigé dans un grec vulgaire tout orné d'archaïsmes maladroits, vers 1550, dans Naples ${ }^{6}$, par un descendant de la famille florentine des Acciaiuoli ${ }^{7}$ qui donna au duché d'Athènes ses derniers maîtres avant les Turcs. Cet auteur, donc, Jean Acciaiuoli, était un homme qui avait servi dans les armées de l'Empereur lors de l'expédition contre Tunis en 1535. Il n'en était pas moins resté profondément grec et orthodoxe. En lui aussi, nous avons le témoin de cette attente, presque messianique, d'un règne universel promis à Charles Quint. C'est même en témoin oculaire de la prise de La Goulette, avant-port de Tunis, qu'il raconte que, pendant l'attaque des Impériaux, à l'intérieur de la citadelle, un eunuque d'origine dalmate y avait libéré les prisonniers chrétiens. Ceux-ci se précipitèrent sur les remparts et

s'écrièrent à pleine voix : "Pour la paix de l'univers, vive l'empereur Charles, sur terre et sur mer ! Subjugue tes ennemis,

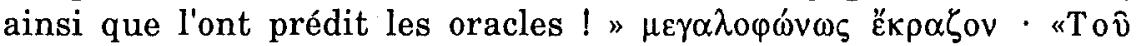

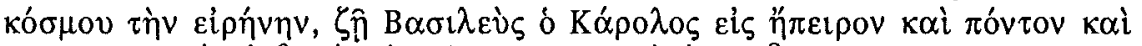

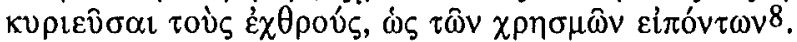

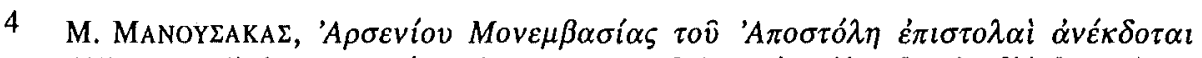

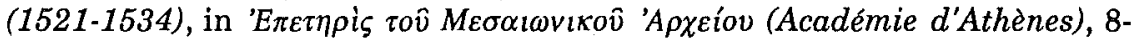
9 (1958-1959), p. 5-56, $\mathrm{n}^{\circ} 11$; complément bibliographique dans ID.,

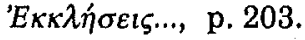

5 M. BataILlon, Mythe et connaissance de la Turquie en Occident au milieu du XVIe siècle, in Actes du Congrès "Venezia e l'Oriente fra Tardo Medievo e Rinascimenton, sous la direction d'A. Tertusi, Venise, 1966, p. 451-470, cite le cas d'un opuscule publié à Venise en 1538 sous le titre Avisi da Constantinopli di cose stupende et meravigliose novamente apparse in quelle parti, con la interpretatione ch'anno fatta gli astrologi et indovini del Gran Turcho circa la ruina sua.

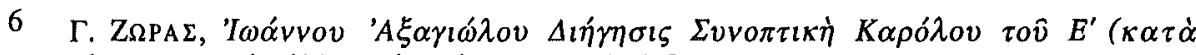

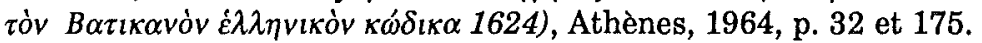

$7 \Gamma$. Zs PAL, ibid., p. 32-34. Cette identification a été contestée, sans raison à mon

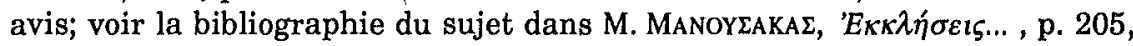
n. 19 et p. 206, n. 20.

8 Jean ACCIAIUOLI, 522-524, texte édité par Г. ZsPAz, op. cit., p. 88. 
Plus loin, l'auteur, s'adressant toujours à Charles Quint, donne deux exemples de ce que pouvaient être ces $\chi \rho \eta \sigma \mu o i ́$ :

Voici les signes qui parurent dans Byzance

- et c'est chose assurée.

La face de lions et de boufs de marbre

regardaient vers le pays d'Orient. Tout à coup,

détournant d'eux-mêmes la tête,

ils regardèrent l'Occident, phénomène surnaturel

puisqu'il est avéré que ces lions-là

étaient inertes - que personne ne vienne dire le contraire!

C'était l'œuvre à l'époque de Sire Léon,

très habile et illustre «lécanomant».

Ils se dressaient au bord du rivage.

On raconte que, deux ans après,

ils se jetèrent d'eux-mêmes là-bas, dans la mer

- où ils se trouvent encore aujourd'hui - sans aucune

intervention humaine.

De même, au milieu de l'illustre, de la grande église consacrée à la très sainte et lumineuse Sagesse Divine, des Turcs prétendent et affirment sous serment qu'ils entendirent, sans voir personne, les psaumes de la Vierge et, particulièrement, ils prétendent qu'en cette terrible journée de la résurrection du Christ, la plus glorieuse de toutes, «Christ est ressuscité d'entre les morts» entendent-ils et

ils en frissonnent.

Voilà ce qu'ils racontent et proclament entre eux.

On fait encore bien d'autres récits à Constantinople

de bouche à oreille et dont tous s'émerveillent.

C'est pour cela que notre foi est restée

fidèle à Notre Seigneur qui ne peut nous tromper.

Ainsi donc, toi qui es le protégé de Dieu, qui as reçu

la bonne nouvelle, qui fus glorifié par Jésus-Christ, magnifique, invincible empereur de la paix, gardien de la foi et de la justice,

il faut te mettre en marche, avec l'aide de Dieu, avec l'armée bénie de ton empire redouté de tous, afin que le peuple de la grande Église d'Orient soit sauvé de la domination des infidèles ${ }^{9}$.

9 Ibid., 1001-1034, p. 100-101:

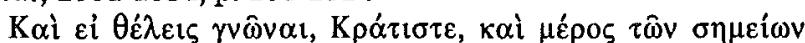

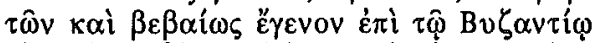

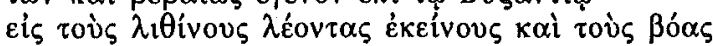

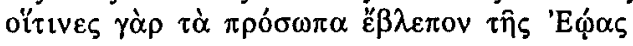


Nous allons voir à quelle tradition se conformait Acciaiuoli en attribuant cet oracle à Léon VI le Sage.

En réalité, c'est par son goût de la rhétorique que ce prince s'est distingué dans l'histoire de Byzance (886-912). C'est lui qui donna à la dynastie macédonienne, qui était de basse origine, le ton de culture qui manquait à son père ${ }^{10}$. De plus, comme juriste, il présida dans ses

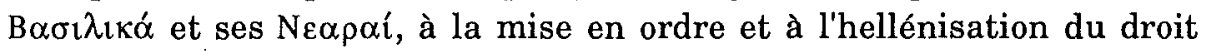
romain ${ }^{11}$. Tout ceci lui valut le surnom de $\Sigma$ ọós, ce qui signifie «Sage» si on veut, mais, dans ce cas-ci, plutôt «Savant». Mais comme, dans les temps qui suivirent, un livre érudit passait facilement pour un grimoire, l'opinion se répandit que la science de ce $\sigma o \varphi o ́ s ~ c o n s i s t a i t$ principalement à lire les destins dans les astres, dans les monuments de Constantinople et même, à en croire Jean Acciaiuoli, dans les

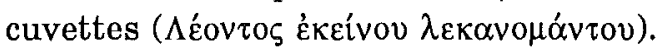

Il est, par ailleurs, hautement improbable que le véritable empereur

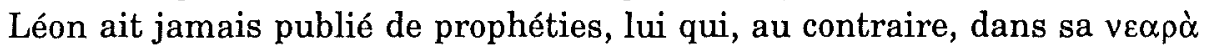

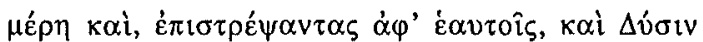

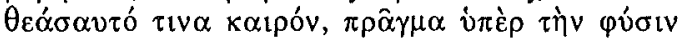

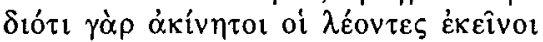

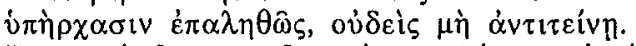

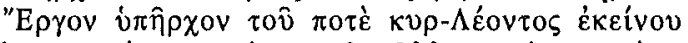

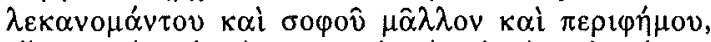

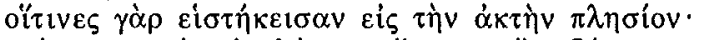

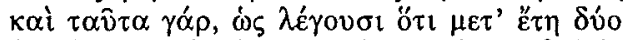

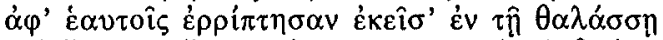

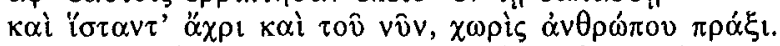

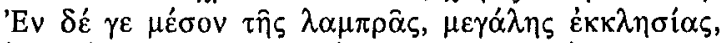

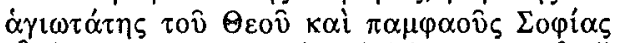

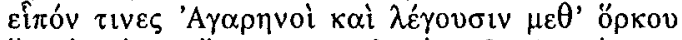

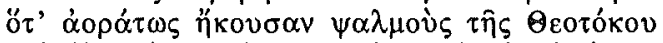

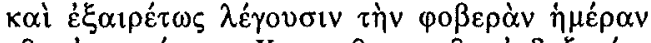

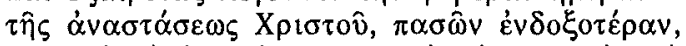

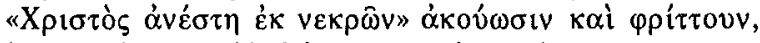

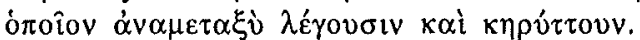

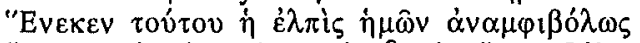

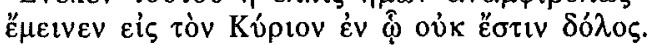

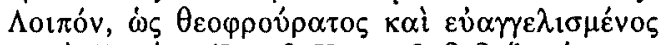

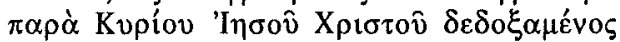

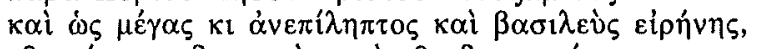

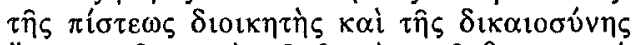

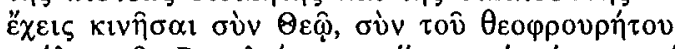

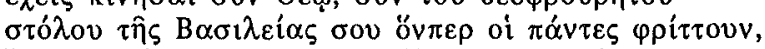

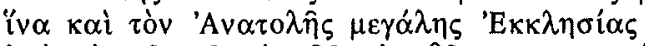

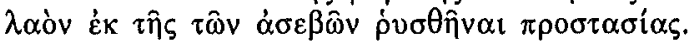

10 P. LEMERLE, Le premier humanisme byzantin, Paris, 1971, p. 206-207.

11 H. MonNIER, Les Novelles de Léon VT le Sage, Paris, 1944. 
65, avait prononcé une sévère condamnation - pouvant aller jusqu'à la peine capitale - contre toute espèce de pratique magique ou divinatoire $^{12}$. Mais voici par quel détour un empereur, qui aimait beaucoup les livres, devint, dans la mémoire de son peuple, une sorte de nécromant.

La colline du Xèrolophos s'élève à l'ouest de la ville de Constantinople, elle domine la Propontide et l'actuel quartier d'Aksaray. Au début du Ve siècle, l'empereur Arcadius et son fils Théodose II y avaient aménagé un forum dont le plus bel ornement était une colonne monumentale comparable à la colonne Trajane ${ }^{13}$. Tout au long d'un fût de trente-cinq mètres de haut, s'enroulait une suite de basreliefs en spirale représentant les événements qui marquèrent l'an 400 sous le règne d'Arcadius, à savoir la défaite des Goths révoltés après leur expulsion de Constantinople; c'est ainsi qu'on devait voir sur les flancs de la colonne, l'armée romaine en armes et la flotte loyaliste bloquant les barbares aux portes de l'Hellespont ${ }^{14}$.

Pendant un temps, cet endroit fut appelé «forum d'Arcadius» ou «colonne d'Arcadius» et, comme le rôle personnel de cet empereur avait été bien insignifiant dans l'histoire, son nom fut bientôt oublié et le quartier reprit son ancien toponyme de "colline desséchée», Xèrolophos. De même, dans l'esprit des Constantinopolitains, s'effaça la signification triomphatrice des bas-reliefs de la colonne.

C'est après un grand malheur qu'on se dit, comme le berger de Virgile, si mens non laeua fuisset : peut-être le ciel nous en avait averti sans qu'on y prenne garde. Au témoignage de Robert de Clari, c'est ce qui est arrivé aux Grecs après la prise de la Ville, en 1204 :

Par dehors de ces colonnes, étaient pourtraites et écrites par prophétie toutes les aventures et toutes les conquêtes qui sont avenues en Constantinople ou qui avenir y devaient. Mais ne pouvait-on savoir l'aventure devant là qu'elle était avenue. Et quand elle était avenue, s'y allaient muser les gens : ils voyaient et apercevaient donc à prime l'aventure. Et cette conquête que les Français conquirent y était écrite et pourtraite et les nefs dont on assaillit par quoi la cité fut

12 C. MANGo, The Legend of Leo the Wise, in Zbornik Radova Vizantološkog Instituta, 6 (1960), p. 59-93, repris dans Byzantium and its Image, Variorum Reprints, Londres, 1984, ch. XVI, p. 60-84, et particulièrement les pages 68-69.

13 R. JANIN, Constantinople byzantine, Paris, 1964², p. 71-72 et 82-84.

14 Sur les représentations de la colonne d'Arcadius, voir J. KolwITZ, Oströmische Plastik der theodosianischen Zeit, Berlin, 1941, p. 17; G.Q. GiLIol, La scilla di bronzo e le altre stratue della spina dell' Ippodromo di Costantinopoli, in ArchClass, 6 (1954), p. 101; G. BECATI, La colona coclide istoriata, Rome, 1960, p. 151. 
prise. Mais ne purent les Grecs savoir devant là que ce fut avenu. Et quand ce fut avenu, on alla regarder et muser en ces colonnes; et trouva-t-on que les lettres qui étaient écrites sur les nefs pourtraites disaient que devers Occident viendrait une gent haut-tondue à côtèles de fer qui Constantinople conquérerait 15 .

Précieux témoignage qui nous montre les bourgeois de Byzance allant s'assurer auprès de la colonne du Xèrolophos que la chute de la capitale de l'univers n'était pas une absurdité, une erreur du destin, mais se trouvait inscrite dans l'ordre des choses. C'est ainsi qu'ils allaient y lire, sur le mode du futur antérieur, l'annonce des événements qui venaient d'arriver : alors les liburnes de la flotte romaine devenaient le présage des galères vénitiennes et la lorique des légionnaires était censée annoncer l'armure des chevaliers francs.

À l'époque de Robert de Clari, ces interprétations de la colonne du Xèrolophos étaient encore anonymes et c'est au cours de ce XIIIe siècle qu'y fut attaché le nom de Léon le Sage. Comme l'histoire véritable de la colonne a été oubliée, on en réinvente une autre, plus significative : la

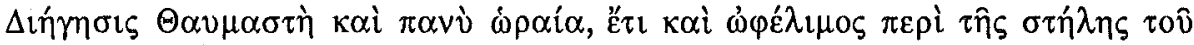
$\Xi \eta \rho \circ \lambda \operatorname{\varphi ́}_{0}{ }^{16}$. Le récit commence par un fait historique : le conflit entre la ville hellénique de Byzance et l'empereur Septime Sévère, de même que le siège que les habitants soutinrent victorieusement pendant trois ans, de 193 à 196. La fiction commence quand il est raconté que c'est l'empereur en personne qui dirigeait les opérations et qu'il avait fixé son camp sur le Xèrolophos précisément, qui se trouvait à l'époque hors les murs. Comme Sévère désespérait de venir à bout de la résistance des Byzantins, une nuit, il interrogea les astres avec l'aide d'un philosophe et astrologue du nom de Jean, pour savoir ce qu'est et ce que deviendra cette mystérieuse cité qui tient en échec Rome elle-même. Alors leur fut révélée la destinée de la future Constantinople et la succession de ses dynasties jusqu'à l'apparition de l'Antéchrist. Et pour que de si troublantes révélations ne soient pas oubliées, Septime Sévère fait dresser la colonne du Xèrolophos où sont sculptées les révélations de l'horoscope, c'est-à-dire toute l'histoire de la Ville jusqu'à la fin des temps. En définitive, Sévère prend la ville, la détruit, en massacre les habitants. Byzance n'est plus qu'un désert jusqu'à ce que Constantin y

15 Robert De Clari, La conquête de Constantinople, XCII, in Historiens et chroniqueurs du Moyen Age, Paris, 1952 (Bibliotheque de la Pléiade), p. 67-68. La tradition associe la colonne du forum Tauri à celle d'Arcadius.

16 Cf. G. DAgron et J. PARAMELle, Récit merveilleux, très beau et profitable sur la colonne du Xerolophos, in T\&MByz, 7 (1979), p. 503-504 et 522-523. 
fonde sa nouvelle capitale dont les murailles enclavent désormais le Xèrolophos et sa colonne. Ainsi se dresse à l'intérieur de la ville un monument énigmatique où sont tracées des images que personne ne comprend. Constantin lui-même et ses successeurs s'interrogent en vain sur la signification des bas-reliefs. C'est en vain qu'on mobilise les philosophes et qu'on les menace de mort s'ils ne parviennent pas à lire ce qu'on devine être des prophéties. Finalement, c'est Léon VI le Sage qui, sans les comprendre vraiment lui-même, les retranscrit dans des oracles en vers iambiques qu'il livre à la postérité ${ }^{17}$.

Cependant, dès les environs de 1300, la Chronique de Morée donne à Léon le Sage un rôle moins passif : nous revenons au mois de février 1204; Alexis Doucas Mov́ $\uparrow \zeta o v \varphi \lambda o \varsigma$ se révolte contre Isaac l'Ange et son fils Alexis IV, représentants du pouvoir installé par les Francs. Ceux-ci réinvestissent Constantinople :

Et quand la ville fut prise, fut aussi pris celui Morchufle, le faux empereur qui avait meurtri son seigneur. Ils l'amenèrent par devant le marquis, le capitaine de l'ost et les autres barons. Et eurent conseil de quelle justice et de quelle mort on ferait mourir celui Morchufle. Adonc vint un sage homme grec, ancien de la cité qui leur dit et conta comment au temps de Léon le Philosophe quand il fut empereur de Constantinople, par son grand sens ordonna et fit moult de chose lesquelles avinrent et aucunes sont à venir à la cité; et entre choses, fit faire un pilier moult grand qui est encore devant l'église de Sainte-Sophie; il la dressa debout et y écrivit des lettres entaillées lesquelles devisent et disent ainsi :

D'ici sera déroupé le faux empereur de Constantinople. Et quand les marquis et autres barons ouïrent et virent les lettres ainsi entaillées, il tombèrent tous d'accord et dirent que, puisque cette prophétie était écrite en ce pilier, il était raison qu'elle fût ores accomplie par ce faux empereur qui avait ainsi faussement meurtri son seigneur. Et lors firent monter celui Morchufle sus le pilier et puis le déroupèrent aval et mourut de telle sorte 18 .

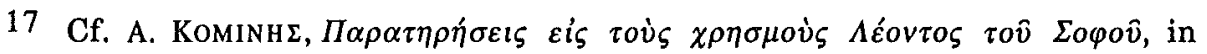
EHBS, 30 (1960-1961), p. 398-412 : essai de synthèse bibliographique.

18 A. Buchon, Recherches historiques sur la Principauté française de Morée et ses hautes baronnies, le livre de la Conqueste de la Princée de Morée, publié pour la première fois d'après un manuscrit de la Bibliothèque des Ducs de Bourgogne à Bruxelles, Paris, 1845, p. 17-18. 
Ici donc, Léon le Sage n'est plus le simple transcripteur du message prophétique, mais il en est l'auteur; bien mieux, il ne se contente pas de prédire l'avenir, il le provoque. C'est ainsi qu'à chacune des chutes de la Ville, en 1204 comme en 1453, nombre de gens étaient persuadés que ces catastrophes avaient été prédites par le savant empereur ${ }^{19}$. Semblablement, on pensait qu'il avait annoncé la défaite des envahisseurs turcs et l'immanquable reconquête de Byzance. Un des oracles avait même désigné par image la puissance libératrice : une nation aux cheveux blonds qui, avec l'alliance des anciens maîtres du pays, mettra

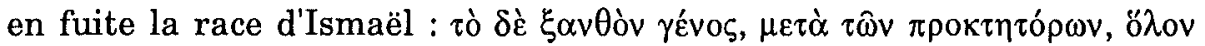

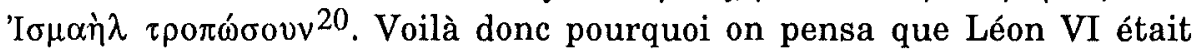

19 Ce sont les conclusions de B. KNös, Les Oracles de Léon le Sage d'après un livre d'Oracles byzantins illustrés, récemment découvert, in 'A

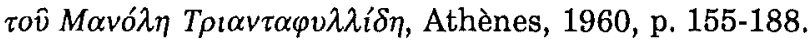

20 C. MANGo, art. cit., p. 78. Ceci peut s'interpréter de diverses façons, ainsi qu'en témoigne Guillaume Postel : «Les Turcs ont davantage en spéciale autorité, et quasi non moindre que leur Alcoran, un Livre de prophéties, là où il est expressément écrit que le prince et le peuple de la jaune couleur doit détruire et tant les Turcs comme tous les Ismaëlites ou Mahumédiques (qui vulgairement se disent mahométans). Et de ceci peut faire foi indubitable, combien que lesdits Turcs cachent autant qu'ils peuvent aux étrangers ladite prophétie. Car il advint qu'étant envoyé pour ambassadeur Monsieur Jean de la Forêt, auvergnat, vers le Grand Turc (et étant avec lui Postel, écrivain de la présente œuvre, pour certaine charge et commandement du roi, qui par ce est témoin digne de foi de ce qu'il écrira), il advint, dis-je, incontinent à la première audience qu'on donna audit de la Forêt, durant l'absence du Seigneur (étant au voyage contre le Sofi), il y eut un des pachas, gouverneur de Constantinople, qui, au lieu de caresser et aimablement recevoir ledit ambassadeur, lui dit qu'il était un espion et un traître qui, non pas pour bien mais comme explorateur du royaume, était venu là. Et pour faire foi certaine qu'il fût ainsi, tira de son sein ledit secret Livre des prophéties (comme s'il eût dû faire foi à l'ambassadeur étant chrétien aussi bien qu'à lui étant turc) et lui commença à dire, présent l'assistance des autres pachas et gouverneurs, comment pour tout certain«Ibn Safran" ou le "fils du jaune» s'entendait entre eux être le "fils des jaunes fleurs de lys» de l'étendart ou écu de France (...) Lors le pauvre ambassadeur, à demi perdu et étonné, voyant que le nier ne lui aidait rien, demanda d'entendre les paroles de ladite prophétie plus à plain. Alors le pacha disant que c'était un prince ou puissance qui avait la plus belle et plus apparente couleur en armes le safran c'est à dire la jaune. Lors l'ambassadeur, sachant comment ils sont grosses gens et tout ignares de cosmographie et beaucoup plus des étranges coutumes, leur dit :

- Oh, pour certain votre prophétie est vraie, mais ce n'est pas le roi de France qui est le Ben Safran, c'est le principal peuple de l'empereur Charles, qui sont les allemands lansquenets et autres dudit pays qui en leurs chausses mi-parties portent la couleur jaune... Guillaume POSTEL, Thrésor des prophéties de 
aussi à l'origine des faits rapportés par Acciaiuoli et que nous connaissons par d'autres sources.

Le palais du Boucoléon à Constantinople était situé au bord de la Propontide. Il tirait son nom d'un groupe de marbre placé sur le quai du port impérial représentant un lion terrassant un taureau ${ }^{21}$. Petro Zen, chroniqueur vénitien, nous rapporte que le 2 novembre 1532, un violent tremblement de terre retourna le monument, en sorte que les deux animaux firent volte-face ${ }^{22}$. Une autre chronique vénitienne, celle De Sagredo, ajoute que la statue qui était placée sur deux colonnes, fut complètement renversée par la suite et précipitée dans la mer ${ }^{23}$.

Voilà donc les faits ${ }^{24}$. Moins d'un an après, voici comment ils étaient interprétés. Mon témoignage est tiré de l'Hodoeporicum Constantinopolitanum, journal de voyage du diplomate flamand Corneille de Schepper qui venait d'arriver à Stamboul au mois de juin 1533:

J'ai été ce jour averti d'un miracle assez notable : il y a une certaine masse de marbre, ici près de la mer, sur laquelle est taillé d'un merveilleux artifice un lion de grande stature tenant un taureau par les cornes; et est ladite masse tant pesante que mille hommes

l'univers, cité en annexe VIII dans G. WEILL, Vie et caractère de Guillaume Postel, thèse latine de 1892 traduite du latin et mise à jour par F. SECRET, Les Belles Lettres, Milan, 1987, p. 253-254.

21 Cf. R. JANIN, op. cit., p. 101.

22 Cité par J. von HAMmer-Purgstall, Histoire de l'Empire Ottoman depuis son origine jusqu'à nos jours, Paris, 1835-1843, III, p. 675.

23 SAGREdo, Memorie istoriche de monarchi ottomani, Venise, 1673, p. 318. La chute du groupe de marbre dans la mer, reprise par Acciaiuoli, confirmait le caractère symbolique de l'accident $:$ le fait divers rejoignait la tradition qui veut qu'à la fin d'un empire, les statues finissent dans les profondeurs de la terre ou des eaux, cf. G. DAGRon, Constantinople imaginaire, études sur le recueil des "Patria», Paris, 1984, p. 145-146 : «Les statues rejoignent les morts; elles sont une population de pierre ou de bronze qui, depuis Constantin, ne se renouvelle plus, et qui par conséquent offre l'image parfaite du viellissement et d'une lente décadence qui conduit la ville à sa fin».

24 Quant à l'autre anecdote rapportée par Acciaiuoli, celle des derviches qui entendirent des voix mystérieuses chanter dans Sainte-Sophie l'Hymne Acathiste et le tropaire de Pâques, elle est citée parmi les événements qui marquèrent l'an 1522, par la Chronique anonyme qui fait suite à l'Histoire

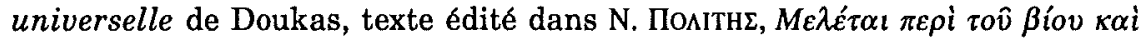

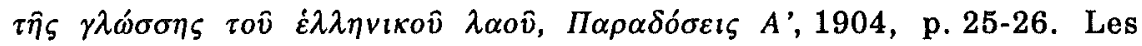
chrétiens y virent l'annonce qu'après la reconquête de Constantinople, les Turcs eux-mêmes entendraient la parole du Seigneur et se convertiraient. 
ensemble ne la pourraient mouvoir; et néanmoins, lorsque le Grand Turc était à la conquête de Hongrie ${ }^{25}$, ledit marbre s'est de soimême tourné de manière que le lion qui avait lors son regard vers l'Asie est maintenant tourné vers l'Europe. Et ils disent que le portier de la porte près laquelle ledit marbre est assis avait été, voulant fermer ladite porte, soudainement saisi de grande frayeur au moyen d'une obscure nuée qui aurait en un instant couvert toutes choses, laquelle, peu après, s'étant retirée, serait ladite masse apparue tournée comme dessus. Ledit marbre était de la même sorte qu'il est maintenant devant que les Turcs prissent Constantinople; mais incontinent qu'ils furent entrés dedans, il s'était tourné, à raison de quoi on estime ledit prodige être fatal, même que ladite ville doive de bref être prise 26 .

Le fait de voir un présage dans le déplacement d'une statue remonte à l'Antiquité. Tacite et Dion Cassius racontent qu'en Germanie une statue de la Victoire qui à l'origine regardait vers la terre ennemie, fut frappée par la foudre et se retrouva tournée vers l'autre côté quasi cederet hostilius. On y vit l'annonce de la défaite de Varus ${ }^{27}$.

En 1550, quand Acciaiuoli écrivit son poème, le souvenir de cet accident était déjà tombé dans le gouffre du passé indistinct où il avait rejoint le mythe de la colonne du Xèrolophos. Le groupe de marbre du Boucoléon devenait lui aussi une œuvre de Léon le Sage disposée tout exprès pour prédire l'avenir. Voilà comment s'écrivent les oracles.

Mais pour une fois qu'une prophétie se risquait à prédire vraiment l'avenir et non pas un fait accompli, elle n'a pas eu de chance. En 1550, Charles Quint avait trop de problèmes de politique intérieure pour songer à la croisade et, pour ses successeurs, la victoire de Lépante fut un coup d'arrêt donné à l'expansionnisme ottoman, mais non une reconquête.

Malgré tous les démentis de l'histoire, on n'en continue pas moins à recopier les Oracles de Léon le Sage parfois dans des manuscrits ornés d'extraordinaires miniatures ${ }^{28}$. À la fin du XVIe siècle et pendant le

25 Du 25 avril au 18 novembre 1532, cf. A. Clot, Soliman le Magnifique, Paris, 1983, p. 116-119.

26 Baron DE SAINT-Genors et G.-A. YSSEL DE SCHEPPER, Missions diplomatiques de Corneille Duplicius de Schepper, in Mémoires de l'Académie Royale des Sciences, de Lettres et des Beaux-Arts de Belgique, 30 (1857), p. 136.

27 TACITE, Annales, XIV, 32; DION CASSIUS, LVI, 24.

28 Le plus remarquable de ces manuscrits est un volume de la Marcienne calligraphié et illustré par Georges Clondzas contemporain et compatriote du

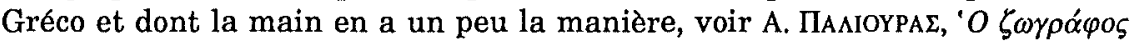

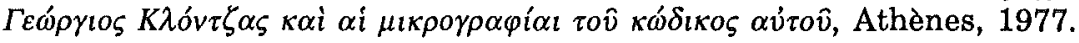


XVIIe, l'imprimerie répandit en Europe occidentale ces Oracles traduits en latin et en langues vulgaires ${ }^{29}$. L'angoisse et l'espérance, plus fortes que l'évidence, en perpétuaient l'autorité. C'est ce que dit bien Michel de Montaigne à qui j'emprunterai ma conclusion :

Je voudrais bien avoir reconnu de mes yeux ces deux merveilles : le livre de Joachim, abbé calabrais, qui prédisait tous les papes futurs et celui de Léon l'Empereur qui prédisait les empereurs et les patriarches de Grèce. Ceci ai-je reconnu de mes yeux : qu'ès confusions publiques, les hommes, étonnés de leur fortune, se vont rejetant (comme à toute superstition) à rechercher au ciel les causes et les menaces de leur malheur 30 .

Rue Sur-la-Fontaine, 114

André DEISSER

B - 4000 LÈGE.

29 Par exemple Vaticinium Severi et Leonis imperatorum, in quo videtur finis Turcarum in praesenti eorum imperatore una cum aliis nonnullis in hac re vaticiniis, latine et italice, Brescia, 1596, avec 16 gravures sur cuivre; Georgii et alterius cuiusdam anonymi excerpta de antiquitatibus Constantinopolitanis, Paris, 1655.

30 Montaigne, Essais, I, XI, Des prognostications. 